\title{
Simultaneous motor and verbal processing of visual information in a modified Stroop test*
}

\author{
HERBERT FRIEDMAN and PETER L. DERKS \\ College of William and Mary, Williamsburg, Virginia 23185
}

\begin{abstract}
Color patches could simultaneously be manually counted and conflicting color words named in the time required for one of the tasks alone. The independence of verbal and nonverbal information processing resembles "split-brain" behavior. Naming color patches while counting color words took more time than would have been required for doing the individual tasks successively, indicating response competition in the standard Stroop test. Form or location stimuli substituted for color words reduced interference when naming color patches.
\end{abstract}

Responding to two dimensions of a multidimensional task is generally difficult. Peterson (1969) has examined several tasks that required concurrent verbal processing, and suggested that, although training can reduce interference, simultaneous processing of material reduces the efficiency of either task. The S may not be able to attend simultaneously to two stimulus inputs (Broadbent, 1958; Treisman, 1969); "central processing capacity" may be used up by the tasks, leaving less capacity for each one individually (Posner, Boies, Eichelman, \& Taylor, 1969; Hammerton, 1969); or covert verbal responses to the task may conflict (Crovitz, Schiffman, \& Rees, 1967).

\section{METHOD}

\section{General Procedure}

Interference in the simultaneous processing of different stimulus dimensions was examined using a modification of the Stroop color-word test (Jensen \& Rohwer, 1966). The usual color-word test requires the $S$ to scan and name the colors of several differently colored inks or patches. If the inks or patches include a conflicting color word (e.g., a red patch with the word "blu" on it), the interference decreases S's rate of naming the patch colors. In this study, only two values were used for each dimension, but $S$ was required to respond simultaneously to both dimensions of a stimulus. For example, with the color-word stimuli, Ss had to read aloud the color words ("red" and "blu") and, at the same time, count the number of red patches and the number of blue patches with two Veeder-Root hand tally counters, one hand for each of the two colors.

\section{Subjects}

Four women and three men, the seven best performers of a group of 32 graduate and undergraduate students, were tested on simultaneously naming and counting the stimuli. The selection procedure allowed the study of maximum capacity rather than typical performance.

*We thank Gary Hudson and Philip Frank for their assistance in collecting and analyzing the data. This work was supported by NASA Grants NGL 47-006-008-FF and NGR 47-006-028.

\section{Materials and Procedure}

The stimuli were 30 red and 30 blue patches of Dymo embossing tape. Each stimulus array was 6 rows of 10 patches in semirandom sequence. Two sets of each array were used, and the arrays were modified after each trial by either interchanging the rows within the array or by changing the array. Stimulus interference with patch color processing was manipulated by using two attributes from one of three other dimensions which were printed in white on the patch. These dimensions and their attributes were color words ("red" and "blu"), forms (XXX and $\mathrm{OOO}$ ), or location (an $\mathrm{X}$ on the right or on the left half of the patch). The two attributes occurred equally often on each color. In a given session, only one of the competing dimensions (words, forms, or location) was employed. Two responses were required to a given array: verbal (naming the two attributes in one of the stimulus dimensions) and manual (counting the two attributes in the other dimension with the two tally counters). For example, $S$ would name the patch colors while manually counting the forms as one task and name the forms while counting the patch colors as another task. Each $S$ was individually tested in a random order of all six possible tasks (i.e., 2 responses $x 3$ competing stimulus dimensions).

On each trial, $S$ scanned the 60 stimulus items, from left to right starting with the top row, naming and counting as fast as he could. A monetary payoff rewarding both speed and accuracy on all tasks permitted as much as $\$ 5$ to be earned in a single session on a given task. Testing continued until $\mathrm{S}$ felt his performance to be asymptotic. Accuracy of the verbal response was verified by the $\mathbf{E}$ during the trial, and accuracy of the counting response was assumed if the final count was correct (i.e., 30 and 30). Errors consistently slowed the response times, and trials with errors were excluded from further analysis. The times required for the two tasks separately, manual counting of one dimension and verbal naming of the other, were obtained in the initial test session and also before and after each combined task session, so that for each $S$ at least four estimates of the response time for each of the six tasks were available. Pilot research has indicated no consistent improvement with practice on the various individual tasks.

\section{RESULTS}

The present study was concerned with maximum capacity. Since random factors such as inattention and confusion would lengthen response times, fastest times for each $S$ rather than average response times for each $S$ were used as measures of performance. 
Table 1

Mean Time Per Item (in Seconds) for Each Single Task Based on Each S's Fastest Errorless Time

\begin{tabular}{lcccc}
\hline & & \multicolumn{3}{c}{ Competing Stimuli } \\
\cline { 3 - 5 } Response & Patch & Words & Form & Location \\
\hline Count & .289 & .351 & .310 & .442 \\
Name & .287 & .302 & .292 & .427 \\
\hline
\end{tabular}

The average over Ss of the errorless fastest time per item on each single task are shown in Table 1. With the exception of location, these rates correspond with the approximately $.300 \mathrm{sec}$ per item reported by Beckwith and Restle $(1066)$ in an earlier study of ouject counting.

All Ss took at least 12 trials on a combined task before terminating the session. To compare the difficulty of the combined tasks, the fastest time without errors in the first 12 trials for each of the six combined tasks for each $\mathrm{S}$ was divided by the sum of his fastest errorless times on the relevant individual tasks to obtain a performance score. For example, if the fastest time for patch counting while naming color words was $.60 \mathrm{sec}$ per stimulus and the fastest time was $.24 \mathrm{sec}$ for patch counting alone and $.36 \mathrm{sec}$ for naming words alone. the score would be $.60 /(.24+.36)=1.0$. A score of 1.0 or greater. as in this example, shows that doing the tasks simultaneously took as long as would have been required for doing the tasks in sequence. A faster combined time of $.36 \mathrm{sec}$ would show that $S$ was processing both dimensions in no more time than was required for the slower of the individual tasks. and would give a score of $.36 /(.24+.36)=.60$. In Fig. 1, the connected points show the mean relative performance for the first 12 trials for each of the six combinations of conditions.

Manually counting, as opposed to naming. the patch color significantly reduces interference between tasks, as shown by the lower relative performance scores $[\mathrm{F}(1,6)$ $=66.39, \mathrm{p}<.001]$. the effect being very large $\left(\mathrm{r}_{\mathrm{m}}>\right.$ 95: Friedman, 1968, 1969). The type of competing stimulus has an effect $[F(2,12)=4.68$, $\left.\mathrm{p}<.05, \mathrm{r}_{\mathrm{m}}>.65\right]$, but the absolute values of the differences are small. The interaction between competing stimulus and response $[F(2,12)=9.67$, $\left.\mathrm{p}<.01, \mathrm{r}_{\mathrm{m}}>.75\right]$ is of more interest and shows the greatest differences between patch-color counting and patch-color naming for the word task, less difference for the form task. and negligible difference for the position task.

The number of trials performed on a given task varied from 12 to over 60 trials. Therefore, to indicate maximum obtained performance for each $\mathrm{S}$, the fastest errorless time on each combined task, regardless of trial, was divided by the sum of the fastest time for the two single tasks. The mean values across $\mathrm{Ss}$ for this measure of maximum capacity are shown in Fig. 1 as unconnected points. There is relatively little improvement after the first 12 trials (showing the effect of prolonged practice to be small), and the general relationship between task and performance is unchanged. This same relationship is also found when the individual S's median times for a task are employed as performance measures. Therefore, the use of fastest times does not distort the relationship.

Table 2 shows, for each of the six tasks, the number of Ss whose fastest time on the combined task was closer to their fastest time on the slower of the single tasks (fast) and the number of Ss whose fastest time was closer to the sum of their fastest times for both single tasks (slow). In other words, "fast" scores indicate that $S$ was closer to simultaneous processing of the two dimensions, while "slow" scores reflect interferefice. Counting the patch color was a much easier task than naming the patch in both the color-word condition (assuming independence of the measurements, $\chi^{2}=$ 7.30. $\left.\mathrm{p}<.01, \mathrm{r}_{\mathrm{m}}>.55\right)$ and the form condition $\left(\chi^{2}=\right.$ 4.57, $\mathrm{p}<.05, \mathrm{r}_{\mathrm{m}}>.45$ ). The two color processing tasks with locations as competing stimuli were equal in difficulty.

Table 2 also denotes the Ss in each condition whose individual task times were equal to or faster than their combined task times (simultaneous processing) and the Ss who failed to get their combined task times below the sum of their individual task times (maximum

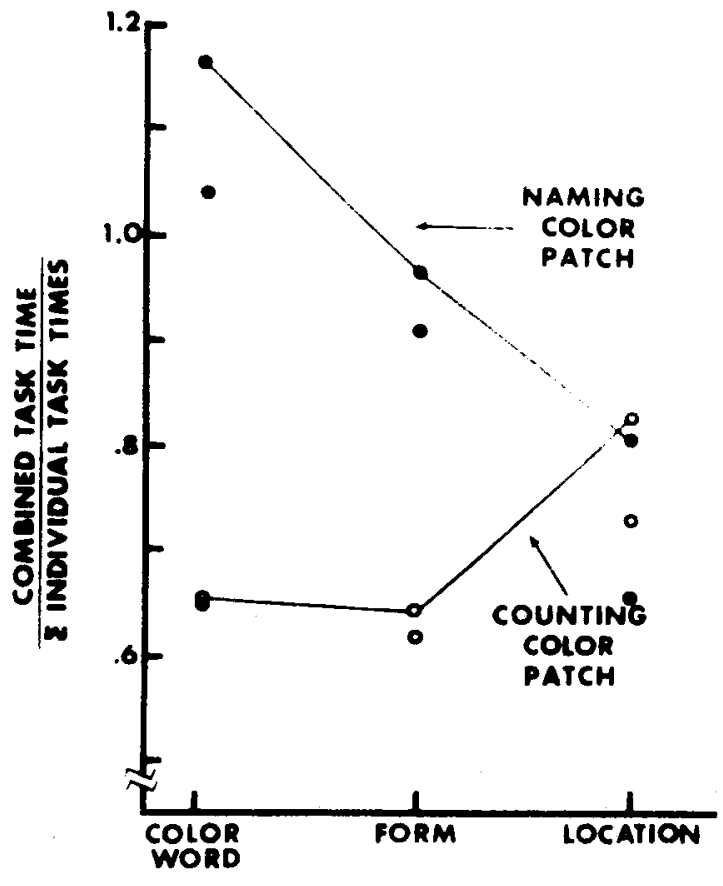

COMPETING STIMULUS

Fig. 1. Mean relative performance as a function of task based only on the fastest errorless trial for each $S$. The connected points were taken from the first 12 trials and represent the data used in the analysis of variance. The unconnected points are based on the fastest times for all trials for each $S$ and represent the data used for Table 2. Counting color patch is shown with open circles and naming color patch with filled circles. 
interference). Colors and words are processed simultaneously only when the patch color is counted manually. If the patch color must be named and the color word counted, it is difficult, and for four of the seven Ss virtually impossible, to complete the dual task in less time than would have been necessary to do the individual tasks successively. This difference in difficulty between two ways of responding to the same two stimulus dimensions shows that the simultaneous processing obtained when the patch color is counted rather than named cannot be explained in terms of Ss simply learning a separate verbal-manual response combination to each of the four stimulus-response combinations.

The possibility that the selection of Ss was responsible for patch counting tasks appearing easier than the patch naming tasks may be ruled out on the basis of a separate test given to two groups of 10 randomly selected Ss. With the combined task time divided by the sum of the individual task times as a measure, the word-name/patch-count group was considerably faster than the word-count/patch-name group, with almost no overlap between the groups $[\mathrm{t}(18)=4.44, \mathrm{p}<.001$, $\left.r_{m}>.70\right]$.

\section{DISCUSSION}

Since the present design requires a separate response to each of two dimensions of a given visual stimulus, the lack of interference of patch color on the word naming task cannot be attributed either to the separation of the stimulus dimensions at the receptor level or to Ss' failing to see or pay attention to the patch color. The ease of the patch-count/word-name task also shows that input and processing capacity must be adequate and interference between the two responses must be minimal. The difficulty of the task when only the response is changed (to patch-name/word-count), therefore, is probably due to response factors, specifically covert verbalization of the word interfering with naming the patch color. Compared to the word stimuli, the form and position stimuli require less verbal processing and, consequently, cause less interference with naming the patch color. In the standard Stroop test, which is a patch color naming task, the slow response times may similarly be due to interference of covert verbal responses to the stimulus word rather than to input or processing limitations (Egeth, Blecker, \& Kamlet, 1964; Murray, Mastronardi, \& Duncan, 1972: Treisman \& Fearnley, 1969).

Processing of verbal and nonverbal information can take place in different cortical hemispheres (Cohen, Noblin, Silverman, \& Penick, 1968). Perhaps Ss can process information simultaneously by responding to different stimulus inputs with separate parts of the brain. If the simultaneous processing shown in this study had been obtained with Ss after surgical removal of the
Table 2

Number of Ss in Each Condition Whose Fastest Errorless Time on the Combined Task was Either Fast (i.e., Closer to the Fastest Errorless Time on the Slower of the Two Individual Tasks) or Slow (i.e., Closer to the Sum of the Individual Task Times)*

\begin{tabular}{llllll}
\hline & & \multicolumn{3}{c}{ Competing Stimuli } \\
\cline { 3 - 6 } & & & Word & Form & Location \\
\hline \multirow{3}{*}{$\begin{array}{lllll}\text { Color-Patch } \\
\text { Response }\end{array}$} & Name & Slow & 7 (iii) & 6 (i) & 2 (i) \\
& & Fast & 0 & 1 & 5 (sss) \\
& Count & Slow & 1 & 1 & 1 \\
& Fast & 6 (ss) & 6 (ssss) & 6 (ss) \\
\hline
\end{tabular}

*The maximum interference (i) and simultaneous processing (s) $S$ s in each condition are shown (see text).

corpus callosum, the ability to complete both tasks in the time required for either one separately might have been at tributed to the dual character of the "split brain" (cf. Gazzaniga, 1967, 1970).

\section{REFERENCES}

Beckwith, M., \& Restle, F. Process of enumeration. Psychological Review, 1966, 73, 437-444.

Broadbent, D. E, Perception and communication. New York: Pergamon Press, 1958.

Cohen, B. D., Noblin, C. D., Silverman, A. J., \& Penick, S. B. Functional asymmetry of the human brain. Science, 1968, $162,475-477$.

Crovitz, H. F., Schiffman, H., \& Rees, J. N. Simulation of mental deficiency: The stupid-reader effect. Psychological Reports, $1967,20,834$.

Egeth, H. E., Blecker, D. L., \& Kamlet, A. S. Verbal interference in a perceptual comparison task. Perception \& Psychophysics, $1969,6,355-356$.

Friedman, H. Magnitude of experimental effect and a table for its rapid estimation. Psychological Bulletin, 1968, 70, 245-251.

Friedman, H. A simplified table for the estimation of magnitude of experimental effect. Psychonomic Science, 1969, 14, 193-195.

Gazzaniga, M. S. The split brain in man. Scientific American, $1967,217,24-29$.

Gazzaniga, M. S. The bisected brain. New York: Appleton-Century-Crofts, 1970.

Hammerton, M. Interference between low information verbal output and a cognitive task. Nature, 1969, 222, 196.

Jensen, A. R., \& Rohwer, W. D., Jr. The Stroop color-word test: A review. Acta Psychologica, 1966, 25, 36-93.

Murray, D. J., Mastronardi, J., \& Duncan, S. Selective attention to "physical" vs "verbal" aspects of colored words. Psychonomic Science, 1972, 26, 305-307.

Peterson, L. R. Concurrent verbal activity. Psychological Review, $1969,76,376-386$.

Posner, M. I., Boies, S. J., Eichelman, W. H., \& Taylor, R. L. Retention of visual and name codes of single letters. Joumal of Experimental Psychology Monograph Supplements, 1969, 79, 1-16.

Treisman, A. M. Strategies and models of selective attention. Psychological Review, 1969, 76, 282-299.

Treisman, A., \& Feamley, S. The Stroop test: Selective attention to colours and words. Nature, 1969, 222, 437-439.

(Received August 14, 1972;

revision received October 4,1972 .) 